Review

\title{
Biology of Metastatic Renal Cell Carcinoma
}

\author{
Michele Milella ${ }^{\bowtie}$, Alessandra Felici \\ Division of Medical Oncology A, Regina Elena national Cancer Institute, Rome Italy
}

$\triangle$ Corresponding author: Michele Milella, MD, Division of Medical Oncology A, Regina Elena National Cancer Institute, Via Elio Chianesi, n. 53, 00144 Rome, Italy. Ph.: +39-06-52666919; Fax: +39-06-52665637; E-mail: milella@ifo.it; michelemilella@hotmail.com

() Ivyspring International Publisher. This is an open-access article distributed under the terms of the Creative Commons License (http://creativecommons.org/ licenses/by-nc-nd/3.0/). Reproduction is permitted for personal, noncommercial use, provided that the article is in whole, unmodified, and properly cited.

Received: 2011.05.26; Accepted: 2011.06.15; Published: 2011.06.16

Abstract

Key words: RCC, Biology, Signal transduction, HIF, mTOR, Angiogenesis

\section{The molecular revolution in renal cell carcinoma}

In recent years, we have witnessed a profound revolution in the way we approach and treat renal cell carcinoma (RCC). In a relatively short time (10 years, approximately), we have gone from a very limited range of therapeutic options [essentially limited to interferon (IFN) and, for the few patients fit enough to receive it, high-dose interleukin-2 (IL-2) or, more recently, allogeneic bone marrow transplant] to almost a plethora of effective agents (at least 6 drugs currently approved for the treatment of advanced RCC and more to come shortly), that has led to define the uncertainties in treatment choices as the 'embarrassment of the riches'. Such a revolution stems from a much improved understanding of RCC biology, predominantly at the cancer cell level, that has led to the recognition of common molecular themes underlying RCC pathogenesis and to the identification of relevant therapeutic targets, such as the activation of pro-angiogenic and of the mammalian target of rapamycin (mTOR) pathways (1). Successful pharmacological targeting of these pathways has dramatically improved the management of patients with metastatic RCC, who have now access to many different treatment choices across multiple lines of treatment, resulting in a striking prolongation of disease control, in most cases compatible with an acceptable quality of life, as well as in a life expectancy that approaches three years from the diagnosis of metastatic disease in the majority of patients. As a consequence, RCC has gone from a 'orphan' disease to a paradigm for the successful development of biology-driven therapies and currently constitutes a unique case among solid tumors, as the management of metastatic disease is entirely based on different classes of molecularly targeted drugs. Exciting successes notwithstanding, several issues remain to be addressed in the treatment of advanced RCC: 1) even in patients who obtain striking clinical responses early in the course of treatment, disease will ultimately escape control and progress to a treatment-resistant state, leading to therapeutic failure; 2) prolonged disease control usually requires 'continuous' treatment, even across different treatment lines, making the impact of chronic, low-grade, toxicities on quality of life greater and precluding, for most patients, the possibility of experiencing 'drug-free holidays'; 3) although we have successfully identified classes of drugs (or molecular mechanisms of action) that are effective in a substantial proportion of patients, we still fall short of molecular predictive factors that identify individual patients who will (or will not) benefit from a specific intervention and still proceed on a trial-and-error ba- 
sis, far from a truly 'personalized' therapeutic approach; 4) finally (and perhaps most importantly), even in the best case scenario, currently available treatments inevitably fail to definitively 'cure' metastatic RCC patients. One of the possible avenues to address such issues, possibly leading to further improvements in the management of advanced RCC, is to better understand the interplay between cancer cell biology, host response, and treatment-induced changes. Indeed, the presence of RCC actually shapes host response (e.g. in terms of immune reaction and microenvironmental changes), which, in turn, influences tumor biology (e.g. in terms of pathway activation), in a complex interplay that is just starting to emerge; in addition, targeted therapies modify both cancer biology and host response, thereby adding a further level of complexity that we are only beginning to appreciate. Unraveling such complex interactions may hold the key to future advances in the comprehension of RCC biology and in the treatment of patients with this disease.

\section{Lessons learned from hereditary syn- dromes and common molecular themes in RCC}

The term RCC encompasses a highly heterogeneous group of malignancies, from both a morphological and a molecular point of view, but emerging evidence indicates that common molecular paths to renal carcinogenesis do exist and may justify, to some extent, shared approaches to the clinical treatment of different RCC subtypes (2). Although most of RCC cases occur in a sporadic form, both clear cell and non-clear cell RCC can occur in the context of inherited cancer syndromes, whose molecular genetics has shed light on potentially common molecular pathogenetic themes (3-4). This is probably best exemplified by von Hippel-Lindau disease (VHL) and tuberous sclerosis (TS), two autosomal dominant inherited syndromes with variable penetrance that carry a high lifetime risk of developing clear cell RCC (5-6). The VHL gene, which targets hypoxia inducible factor (HIF)-1 $\alpha$ for degradation by the proteasome, is mutated or silenced in up to $75 \%$ of sporadic clear cell RCC, suggesting that genetic abnormalities involved in inherited RCC syndromes (and subsequent alterations in downstream intracellular signaling cascades) may also play a central role in sporadic RCC. In tumors carrying a mutated VHL, increased levels of HIF-1 $\alpha$ play a critical oncogenic role by stimulating the transcription of many crucial downstream effectors, including vascular endothelial growth factor (VEGF), platelet-derived growth factor (PDGF),
c-Met, transforming growth factor (TGF)- $\alpha$, and the stromal-derived factor (SDF)-1/CXCR4 ligand/receptor pair, among others (7). Of note, signaling pathways initiated by such effectors, VEGF and PDGF in particular, are the therapeutic targets of monoclonal antibodies and small-molecule kinase inhibitors (TKIs) that currently constitute the mainstay of clinical RCC treatment. TS, on the other hand, is an autosomal dominant disorder with $95 \%$ penetrance, caused by mutations in either the TSC1 (9q34) or the TSC2 (16p13.3) genes, encoding for the hamartin and tuberin proteins, respectively. Hamartin and tuberin physically interact to form a complex, which, through the GAP activity of tuberin, inactivates the small G-protein Rheb, thereby relieving Rheb-mediated mTOR inhibition. Therefore, genetic inactivation of TSC1/2 results in the uncontrolled activation of the mTOR pathway, leading, among other effects, to increased synthesis and accumulation of HIF, even in the absence of hypoxia, and transcription of HIF-dependent genes (8-9). Indeed, activated mTOR, may exacerbate the loss of VHL function (or the effects of hypoxia) by further elevating HIF-1 through increased translation, thus providing a direct link between the HIF/angiogenesis and the mTOR paths to renal carcinogenesis. Because unregulated angiogenesis is a prominent feature of RCC, the inhibition of mTOR is relevant clinically and may inhibit angiogenesis through a mechanistic approach that differs from that of VEGF receptor-targeted agents. In addition to clear cell RCC, the spectrum of renal manifestations in TS also includes development of multiple angiomyolipomas, renal cysts, and non-clear cell RCC (papillary and chromophobe carcinomas). Other hereditary RCC syndromes involving non-clear cell RCC have also been identified and characterized in terms of the underlying genetic lesions; interestingly, increased HIF expression and transcriptional activity activation of the mTOR pathway both appear to be central to the development of different renal manifestations of disease, including benign (angiomyolipomas, renal cysts, oncocytomas), borderline (hybrid oncocytic tumors), and frankly malignant (papillary and chromophobe RCC) lesions (10). Genetic aberrations in TSC1/2 and Birt-Hogg-Dubè (BHD) directly impinge on the activation of the mTOR pathway, leading to the development of an array of renal lesions that can be partially reversed by rapamycin-mediated inhibition of mTOR, both in preclinical models and human patients with TSC; in the highly aggressive papillary type 2 RCC observed in hereditary leiomyomatosis and renal cell cancer (HLRCC), fumarate hydratase (FH) deficiency creates a pseudohypoxic intracellular environment (9), lead- 
ing to HIF accumulation; from a molecular standpoint, this situation is similar to that observed in VHL mutant RCC cells, where HIF- $1 \alpha$ translation and accumulation can be prevented by mTOR inhibition, thereby rendering HIF-overexpressing cells particularly prone to the growth inhibitory effects of mTOR inhibitors, both in vitro and in vivo. More recently, computational analysis of gene expression data derived from papillary RCC revealed that a transcriptional signature indicative of MYC pathway activation is present in high-grade type 2 papillary RCC. The MYC signature was associated with amplification of chromosome $8 \mathrm{q}$ and overexpression of MYC that maps to chromosome 8q24 and, reflective of the association of an active MYC signature component with papillary type 2 , the presence of this pathway signature component was also associated with a highly aggressive clinical behavior and poor overall survival (11). Recent evidence indicates the existence of an important growth-regulatory crosstalk between the MYC, HIF, and the mTOR pathway: indeed, both HIF-1 and HIF-2 may directly or indirectly control MYC activity, on one hand, and tuberin loss may de-repress MYC protein, on the other, positioning the connection between these two growth regulators to act as a feed-forward loop that would amplify the oncogenic effects of decreased tuberin or increased MYC expression (12). Overall, both genetic and molecular data strongly indicate that common avenues do exist in renal carcinogenesis and that HIF accumulation and mTOR activation represent common molecular themes across a spectrum of both benign renal lesions and different RCC subtypes, including both clear cell and non-clear cell forms, and thus constitute widespread therapeutic targets in both sporadic and familial RCC.

\section{Resistance and escape mechanisms}

Understanding (and overcoming) primary and/or acquired resistance to both HIF/VEGF- and mTOR-targeted agents is perhaps the most important issue to address, in order to make further clinical progresses in the management of advanced RCC. While the molecular mechanisms of primary resistance are still elusive, clues to the development of acquired resistance in patients that initially respond favorably to VEGF/VEGF receptor-targeted agents are starting to emerge (13-14). First, the pathway may be incompletely blocked, due to an intrinsically low potency of the agent employed as first-line or to an adaptive response that leads to increased drug metabolism/extrusion, with consequently reduced effective drug levels, or to increased signaling through the same ligand/receptor pairs that are being targeted (in most cases VEGF/VEGFRs and PDGF/PDGFRs); such a resistance mechanism would explain the clinical finding of incomplete cross-resistance between VEGF/VEGFR-targeted drugs, particularly when crossing over to a second-line agent more potently targeting the same pathway. Second, under the selective pressure of prolonged treatment with anti-angiogenic agents (mostly targeting the VEGF axis), RCC may ultimately resume an angiogenic state either by increasing HIF-mediated transcription of VEGF and PDGF or through alternative, non HIF-mediated pro-angiogenic factors, such as fibroblast growth factor (FGF), interleukin-8 (IL-8), placental-derived growth factor (PlGF), angiopoetins, etc.; in these cases, the tumor would still depend on its ability to stimulate angiogenesis, thus targeting HIF directly or the alternate angiogenic pathway(s) in sequence could still lead to disease control. Third, RCC cells may adapt to an intrinsically hypoxic, anti-angiogenic, microenvironment by activating intracellular signaling pathways, such as the mTOR pathway, that would help them coping with a state of high metabolic stress, while maintaining their ability to grow; the development of resistance through this molecular mechanism actually constitutes the rationale for sequential (or alternating) VEGF/mTOR pathway targeting, that is becoming increasingly popular in the clinical setting (see below).

In addition to cancer cell-centered mechanisms of resistance, a potentially important and relatively unexplored area of research is the contribution of host-derived microenvironment to the ability of tumors to adapt to prolonged blockade of VEGF-mediated angiogenesis and escape from anti-angiogenic drug mediated growth control (15). For example, increased host-derived pericyte coverage and recruitment of bone marrow-derived cell populations (that are 'normal' in nature) may protect tumor blood vessels and endorse restored neo-vascularization, even in the presence of continuous VEGF blockade; interestingly, activity of the HIF family of transcription factors appears to play a crucial role not only in the biology of the RCC cell itself, but also in orchestrating the response of host-derived cells to prolonged anti-angiogenesis, thereby constituting a potential therapeutic target whose direct inhibition may simultaneously hit both the cancer cell and the surrounding microenvironment. Finally, in the presence of a hostile, anti-angiogenic, microenvironment the RCC cell may acquire a more motile, invasive, phenotype that allows them escaping nutrient and oxygen deprivation by metastasizing to different organs where they could hijack host-derived protective mechanisms to create a more favorable 
environment (16). Studies conducted in tumors that have become resistant to sunitinib-mediated anti-angiogenesis, indeed, indicate that resistant tumor cells acquire a 'sarcoma-like' phenotype, with decreased cytokeratin and increased vimentin expression (indicative of an epithelial-to-mesenchymal transition - EMT), invade surrounding tissues, and display decreased vascular density (17).

\section{Rationale for sequencing agents with dif- ferent mechanisms of action}

Metabolic adaptation to stress conditions is an important mechanism involved in tumor progression and development of resistance mechanisms. A solid tumor can outstrip its nutrient and oxygen supply as it grows, resulting in metabolic stress. As a consequence, tumor cells must undergo a period of metabolic adaptation to survive this stress or undergo apoptosis; angiogenesis and neovascularization is one strategy of metabolic adaptation used by tumors to relieve this stress, while maintaining their capacity to grow indefinitely (18). By blocking angiogenesis for prolonged periods of time, as it is usually the case for the first-line treatment of metastatic RCC, the tumor is put back in a state of metabolic stress, to cope with which it must activate alternative relief mechanisms. Given its strategic position in the regulation of cell growth, metabolism, and angiogenesis, the mTOR pathway is a likely candidate to be a crucial regulator of 'metabolic adaptation' in situations in which the VEGF-based angiogenic switch is pharmacologically inhibited. Indeed, mTOR senses availability of aminoacids, metabolic fuel, and energy (essential for protein synthesis, cell growth, proliferation, and survival) and its activation supports growth and survival by increasing cell access to nutrients and metabolic fuels, through increased expression of nutrient transporters. It is, therefore, reasonable to speculate that alternating agents with different modes of action would actually turn tumor's ability to activate either neo-angiogenesis or mTOR signaling to cope with situations of metabolic stress to our advantage: indeed, after prolonged exposure to VEGF/VEGFR-targeted agents, RCC cells may activate $\mathrm{MTOR}$ to cope with metabolic stress and survive; if mTOR is blocked at this stage, tumor cells would be most sensitive to its action and would go back to a neo-angiogenesis driven response to metabolic stress, again becoming sensitive to VEGF-targeted strategies. Although such hypothesis remains to be tested, both experimentally and clinically, solid clinical evidence indicates that mTOR inhibitors are effective after progression to VEGF/VEGFR-targeted strategies and initial reports suggest that rechallenge with a VEGFR
TKI (even the same agent used as first-line) after an mTOR inhibitor may lead to substantial clinical benefit.

\section{The importance of the 'host'}

Another dimension, that is only now starting to emerge from the literature, is the complex interplay between the cancer cell(s), the host immune system, and the molecularly targeted agents that are used for treatment. It is relatively well known that the presence of a tumor may shape host immune response by creating an immunosuppressive environment; in the specific case of RCC, which is characterized by prominent activation of the HIF/VEGF axis, VEGF itself and/or other cytokines produced by tumor cells exert profound immunosuppressive effects by impairing dendritic cell maturation from myeloid progenitors, increasing Treg-mediated suppression of T-cell responses, and shifting the balance towards a Th2 type of response with secondary production of IL-10, IL-4, and IL-6. Not only tumors, including RCC, may effectively suppress tumor-specific immune responses, but they can also hijack host-derived cell populations, turning them into powerful allies that help creating a pro-angiogenic, tumor-promoting microenvironment (19): indeed, tumor-infiltrating macrophages, neutrophils, dendritic cells (DCs), and natural killer cells may shift from the production of anti-angiogenic/tumor suppressing cytokines, such as IFN- $\gamma$ and IL-12, to the secretion of VEGF, PIGF, FGF, PDGF, IL-8, matrix metalloproteases (MMPs), cyclooxygenase-2 (Cox-2), and arginase; moreover, in the tumor microenvironment myeloid progenitor cells may be forced to differentiate into myeloid-derived stromal cells (MDSCs), that potently support tumor-driven vasculogenesis and help protecting tumor-associated neo-vessels from anti-angiogenic therapeutic attack.

The immune-modulatory effects of therapeutic agents (mostly VEGF/VEGFR and mTOR inhibitors) commonly used to treat RCC are only beginning to surface (20). For example, two commonly used anti-angiogenic multi-kinase inhibitors, such as sorafenib and sunitinib, clearly differ in their ability to, directly or indirectly, influence immune response: indeed, sorafenib appears to impair, while sunitinib stimulates, terminal DC maturation and ability to co-stimulate T-cell responses; sunitinib, but not sorafenib, inhibits MDSC immune-suppressive activity, reduces both MDSC and Treg circulating numbers, and corrects Th2 bias. It would be even more interesting to understand the immunomodulatory effects of rapamycin derivatives, such as temsirolimus and everolimus, in the specific context of RCC treatment, 
considering their well-established alternative use as immunosuppressants in organ transplantation.

\section{Summary and conclusions}

In the past ten years we have made exceptional progresses in the understanding of RCC biology, particularly by recognizing the crucial pathogenetic role of activation of the HIF/VEGF and mTOR pathways. This has resulted in the successful clinical development of anti-angiogenic and mTOR-targeted drugs, which have profoundly impacted on the natural history of the disease and have improved the duration and quality of RCC patient lives. However, further improvements are still greatly needed, particularly in the quest for 'curative' treatments, currently lacking in the setting of advanced RCC. As highlighted above, unraveling the complex mechanisms by which RCC shapes host microenvironment and immune response and therapeutic treatments, in turn, shape both cancer cell biology and tumor-host interactions may hold the key to future advances in such a complex and challenging disease.

\section{Conflict of Interest}

The authors have declared that no conflict of interest exists.

\section{References}

1. Rini BI. Many treatment options, one patient. J Clin Oncol. 2009; 27: 3225-34.

2. Linehan WM, Bratslavsky G, Pinto PA, et al. Molecular diagnosis and therapy of kidney cancer. Annu Rev Med. 2010; 61: 329-43.

3. Linehan WM, Pinto PA, Srinivasan R, et al. Identification of the genes for kidney cancer: Opportunity for disease-specific targeted therapeutics. Clin Cancer Res. 2007; 13: 671s-9s.

4. Linehan WM, Pinto PA, Bratslavsky G, et al. Hereditary kidney cancer: unique opportunity for disease-based therapy. Cancer. 2009; 115 (10 suppl): 2252-61.

5. Maher ER. Von Hippel Lindau disease. Curr Mol Med. 2004; 4: 833-42.

6. Henske EP. The genetic basis of kidney cancer: Why is tuberous sclerosis complex often overlooked? Curr Mol Med. 2004; 4: 825-31.

7. Kaelin WG Jr. The von Hippel-Lindau tumor suppressor protein and clear cell renal carcinoma. Clin Cancer Res. 2007; 13: 680s-4s.

8. Yeung RS. Lessons from the Eker rat model: From cage to bedside. Curr Mol Med. 2004; 4: 799-806.

9. Cook JD, Walker GL. The Eker rat: Establishing a genetic paradigm linking renal cell carcinoma and uterine leiomyoma. Curr Mol Med. 2004; 4: 813-24.

10. Ciuffreda L, Di Sanza C, Cesta Incani U, et al. The mTOR pathway: A new target in cancer therapy. Curr Cancer Drug Targets. 2010; 10: 484-95.

11. Furge KA, Chen J, Koeman J, et al. Detection of DNA copy number changes and oncogenic signaling abnormalities from gene expression data reveals MYC activation in high-grade papillary renal cell carcinoma. Cancer Res. 2007; 67: 3171-6.
12. Lendahl U, Lee KL, Yang H, et al. Generating specificity and diversity in the transcriptional response to hypoxia. Nat Rev Genetics. 2009; 10: 821-32.

13. Rini BI, Atkins MB. Resistance to targeted therapy in renal-cell carcinoma. Lancet Oncol. 2009; 10: 992-1000.

14. Rini BI. New strategies in kidney cancer: Therapeutic advances through understanding the molecular basis of response and resistance. Clin Cancer Res. 2010; 16: 1348-54.

15. Bergers G, Hanahan D. Modes of resistance to anti-angiogenic therapy. Nat Rev Cancer. 2008; 8: 592-603.

16. Paez-Ribes M, Allen E, Hudock J, et al. Antiangiogenic therapy elicits malignant progression of tumors to increased local invasion and distant metastasis. Cancer Cell. 2009; 15: 220-31.

17. Faivre S, Demetri G, Sargent W, et al. Molecular basis for sunitinib efficacy and future clinical development. Nat Rev Drug Discov. 2007; 6: 734-45.

18. Jones RG, Thompson CB. Tumor suppressors and cell metabolism: A recipe for cancer growth. Genes Dev. 2009; 23: 537-48.

19. Noonan DM, De Lerma Barbaro A, Vannini N, et al. Inflammation, inflammatory cells, and angiogenesis: Decisions and indecisions. Cancer Metastasis Rev. 2008; 27: 31-40.

20. Seliger B, Massa C. Rini B, et al. Antitumour and immune-adjuvant activities of protein-tyrosine kinase inhibitors. Trends Mol Med. 2010; 16: 184-92. 